\title{
SABERES E DESAFIOS NA RESIDÊNCIA MULTIPROFISSIONAL EM SAÚDE SOB A VISÃO DE RESIDENTES ${ }^{1}$
}

\author{
KNOWLEDGE AND CHALLENGES IN MULTIPROFESSIONAL RESIDENCE IN \\ HEALTH FROM THE RESIDENTS POINT OF VIEWI
}

\section{Fernanda Lencina de Amarante ${ }^{2}$, Stefani Gabriela da Silva dos Santos ${ }^{3}$, Ana Paula Brutti Linhatti ${ }^{4}$, Suani Feltrin da Silva ${ }^{5}$, Joyce Nagera Braz ${ }^{6}$ e Luciana Cezimbra Weis ${ }^{7}$}

\section{RESUMO}

Introdução: Este estudo apresenta os desafios da residência multiprofissional em saúde sob a visão das residentes e sua orientadora fisioterapeuta. Objetivo: socializar saberes e desafios vivenciados no campo de atuação da residência. Metodologia: o presente estudo trata-se de uma abordagem descritiva, caracterizado como um relato de experiência das residentes em Reabilitação Física, correspondendo à atuação em campo pelo período de março a novembro de 2020. Compreende os núcleos profissionais de Enfermagem, Fisioterapia, Nutrição, Psicologia e Terapia Ocupacional, atuantes em um hospital gaúcho de baixa e média complexidade em turno integral. Conclusão: a troca de saberes e experiências entre as residentes prevaleceu frente às práticas, mediante as dificuldades encontradas, possibilitando um olhar integral e humanizado para pacientes e as próprias profissionais. O senso crítico é primordial diante de desafios encontrados na atuação em etapa formativa, vislumbrando maior preparação no desempenho profissional futuro. Contribuindo para uma experiência primordial no mercado de trabalho.

Palavras-chave: Equipe Multiprofissional, Internato e Residência, Prática Profissional, Profissional da Saúde.

\section{ABSTRACT}

This study presents the challenges of multiprofessional residency in health from the perspective of residents and her physical therapist advisor. Objective: socialize knowledge and challenges experienced in the Residence's field of action. Methodology: the present study is a descriptive approach, characterized as a report of the residents' experience in Physical Rehabilitation, corresponding to the performance from March to November 2020. It comprises the professional cores of Nursing, Physiotherapy, Nutrition, Psychology and Occupational Therapy, working in a hospital gaucho of low and medium complexity full-time. Conclusion: The exchange of knowledge and experiences among the residents prevailed before the practices, due to the difficulties encountered, allowing an integral and humanized look for patients and the professionals themselves. Critical

11 Trabalho de Residência Multiprofissional em Saúde.

2 Autor, Terapeuta Ocupacional, Especialista em Sistema Público de Saúde pela Universidade Federal de Santa Maria (UFSM), Residente em Reabilitação Física - Universidade Franciscana (UFN). E-mail: amarante.fer@gmail.com 3 Coautor, Psicóloga, Residente em Reabilitação Física - Universidade Franciscana (UFN). E-mail: sgsssantos@ucs.br 4 Colaborador, Fisioterapeuta, Residente em Reabilitação Física - Universidade Franciscana (UFN). E-mail: ana.paula. brutti@hotmail.com

5 Colaborador, Nutricionista, Especialista em Nutrição Clínica e Metabolismo - Colégio Brasileiro de Estudos Sistêmicos (CBES), Residente em Reabilitação Física - Universidade Franciscana (UFN). E-mail: suani.feltrins@gmail.com

6 Colaborador, Enfermeira, Especialista em Enfermagem no Trabalho - Uninter, Residente em Reabilitação Física Universidade Franciscana (UFN). E-mail: joycenagera@gmail.com

7 Orientador, Fisioterapeuta, Mestre em Fisioterapia pela Universidade Metodista de Piracicaba, Docente no Programa de Residência Multiprofissional em Reabilitação Física - Universidade Franciscana (UFN). E-mail: lucianaweis@ufn.edu.br 
sense is paramount in the face of challenges encountered in acting in a formative stage, envisioning greater preparation for future professional performance. Contributing to a primordial experience in the job market.

Keywords: Patient Care Team, Internship and Residency, Professional Practice, Health Personnel.

\section{INTRODUÇÃO}

A Residência Multiprofissional em Saúde (RMS) se caracteriza como uma formação em área da saúde em nível de especialização, onde é realizada através do trabalho em saúde. Através de ações de Educação Permanente em Saúde (EPS) desenvolvidas na RMS, desenvolveu-se um espaço para gerar mudanças aspiradas pelos profissionais de saúde com a finalidade de consolidar os princípios do Sistema Único de Saúde (SUS) (SILVA et al., 2016).

Os Programas de Residência Multiprofissional em Saúde têm carga horária total de 5.760 horas, possuindo a intenção de possibilitar aprendizado necessário para o caráter de especialização. Estas horas são distribuídas em atividades práticas e teórico-práticas, correspondendo a $80 \%$ da carga horária total e, em atividades teóricas, 20\% (COMISSÃO NACIONAL DE RESIDÊNCIA MULTIPROFISSIONAL EM SAÚDE, 2014). A avaliação do residente é do tipo formativa, de formato contínuo, desenvolvida durante todo o processo de ensino e aprendizagem, e do tipo somativa, para compreender a evolução do residente no alcance dos objetivos esperados para a certificação e notas finais semestralmente (ANASTASIOU; ALVES, 2003).

É proposta dos programas de residência que façam parte do processo a formação em serviço a supervisão direta por profissionais denominados preceptores, a supervisão acadêmica como tutoria, regime de dedicação exclusiva, cenários de atuação em serviços da rede de atenção à saúde (SILVA; GOMES, 2018). Ademais, ao término do programa, o Profissional de Saúde Residente deverá apresentar individualmente trabalho de conclusão de Residência (COMISSÃO NACIONAL DE RESIDÊNCIA MULTIPROFISSIONAL EM SAÚDE, 2014).

A partir das necessidades e realidades locais e regionais, vivenciadas na rotina do SUS, as residências multiprofissionais e em área profissional da saúde foram criadas segundo a Lei $\mathrm{n}^{\circ} 11.129$ de 2005 (BRASIL, 2005), orientada pelos princípios e diretrizes do SUS, abrangendo os núcleos profissionais: Educação Física, Odontologia, Biomedicina, Enfermagem, Psicologia, Serviço Social, Nutrição, Fisioterapia, Terapia Ocupacional, Ciências Biológicas, Farmácia, Fonoaudiologia e Medicina Veterinária (SILVA; MOREIRA, 2019). Valorizam-se os profissionais da saúde através de estímulos e acompanhando constantemente a Política de Educação Permanente em Saúde, incentivando a qualificação e a formação das residências, juntamente com as distintas esferas de governo (MARTINS et al., 2016).

Vivenciar no processo de formação a Educação Interprofissional em Saúde possibilita que o profissional se torne crítico e reflexivo, mais integrado à equipe, envolvido com as ações de saúde, possibilitando uma visão mais ampla e sensível para o contexto familiar, cultural e socioeconômico 
do paciente. A prática colaborativa permite que os residentes tenham um aprendizado com o olhar no coletivo e no trabalho em equipe, sendo possível a integralidade do cuidado (ROSSIT et al., 2014). A integralidade dentro de um programa de residência considera o conceito ampliado de saúde em relação ao ambiente cultural, social e político dos usuários, o atendimento deve suprir as necessidades múltiplas de saúde, através do trabalho coletivo e ações interdisciplinares (SILVA, 2018).

O objetivo do Programa de Residência Multiprofissional em Reabilitação Física desta Instituição de Ensino Superior é qualificar profissionais para atuarem em reabilitação através de práticas multiprofissionais e interdisciplinares. Contribuindo para o aperfeiçoamento ético, técnico-científico e humano para que o atendimento integral à saúde da população e dos sujeitos, na rede de cuidados à pessoa com deficiência ocorra, possibilitando a integração dos distintos serviços da rede de reabilitação, sistematizando os diferentes níveis de complexidade no atendimento à pessoa com necessidades ortopédicas, neurológicas e traumatológicas (UFN, 2015).

Sendo assim, a busca por trabalhos - em plataformas científicas - que retratam a realidade do residente esteve presente na rotina das profissionais em questão. O que foi encontrado foram relatos de experiência abordando a prática enquanto profissional da saúde em suas atuações específicas de núcleo. Logo, ao ser percebida uma escassez de publicações que abordam a práxis sob a ótica do residente em seu processo de formação, constatou-se a necessidade e coerência da inclusão e validação do ponto de vista único e subjetivo despertado pela experiência que estes profissionais vivenciam na sua formação enquanto atuantes e constitutivos de um programa de Residência. Além de que, são vislumbrados os benefícios que a explanação de trocas de experiências pode propiciar, uma vez que socializar saberes e desafios vivenciados no campo de atuação da residência é o objetivo deste estudo.

\section{MÉTODOS}

O presente estudo possui uma abordagem descritiva, caracterizado como um relato de experiência. O qual será desenvolvido a apartir das vivências, observações e reflexões de residentes em Reabilitação Física no primeiro ano da especialização. Compreende os núcleos profissionais de Enfermagem, Fisioterapia, Nutrição, Psicologia e Terapia Ocupacional, atuantes em um hospital de média complexidade na região central do estado. A atuação no campo de prática foi durante o período de março a novembro de 2020, em turno integral, de segunda a sexta-feira. A parte teórica ocorreu no turno da noite, totalizando dezesseis disciplinas cursadas e finalizadas.

Devido ao momento atual de pandemia, algumas alterações na grade curricular da Residência tiveram que ser feitas. Com isso, repercutiu no formato e distribuição das atividades práticas do programa. Dessa forma, ao invés das profissionais atuarem no hospital e no Laboratório de Ensino Prático - nos moldes ambulatoriais - disponibilizado pela instituição de ensino, a atuação se deu apenas na área hospitalar. 
As informações salientadas ao decorrer do escrito, tiveram como respaldo a realidade vivenciada pelos profissionais neste excepcional novo formato de desenvolvimento do programa de Residência Multiprofissional com Ênfase em Reabilitação Física. Este também segue os preceitos éticos que constam na Resolução n 510 do Conselho Nacional de Saúde (BRASIL, 2016), que dispõe sobre pesquisas em Ciências Humanas e Sociais, qual dispensa a obrigatoriedade de registro no CEP/ CONEP para divulgação de atividade realizada com o intuito exclusivamente de educação, ensino ou treinamento sem finalidade de pesquisa científica, reiterando ao propósito deste relato.

\section{RESULTADOS E DISCUSSÃO}

\section{O CAMPO DE ATUAÇÃO}

A equipe de residentes é formada por cinco profissionais, sendo enfermeira, fisioterapeuta, nutricionista, psicóloga e terapeuta ocupacional. A atuação prática das mesmas se dá de modo integral em uma unidade clínica de um hospital de média complexidade no interior do Rio Grande do Sul, de segunda-feira a sexta-feira.

A prática das residentes, de modo sucinto, se concentra em atendimentos nos leitos de internações de modo individual ou compartilhado. Também integram os rounds multiprofissionais onde são discutidos os planos de tratamento dos pacientes junto à equipe da Comissão de Controle de Infecção Hospitalar (CCIH) e assistência - médicos, enfermeiros, fisioterapeutas, nutricionistas, psicólogos, farmacêuticos, assistente social. Por vezes, sob apoio dos respectivos preceptores, realizam capacitações aos colaboradores do hospital, sendo estas planejadas pelas residentes e protocoladas junto ao Plano de Capacitações da Unidade em que atuam.

Outra atividade desenvolvida pelas profissionais, em conjunto com as residentes da ênfase em Infectologia e Neurologia, são as referências e contrarreferências, que são os encaminhamentos dos pacientes para a continuidade de cuidados após a alta hospitalar seja em ambulatório especializado ou para o acompanhamento na Atenção Básica. As residentes também participam ativamente dos comitês do hospital como a Comissão de Educação Permanente e Continuada em Saúde e a Comissão de Grupo de Trabalho Humanizado, propondo, pactuando e executando treinamentos e outras ações estratégicas dentre as demandas específicas de cada comitê.

Ademais, as residentes atuam em um projeto voltado à saúde do trabalhador do referido hospital, onde interdisciplinarmente realizam uma escuta ativa e humanizada além de lançar mão de técnicas próprias de cada núcleo profissional para orientações e atendimentos individuais. Neste sentido, também são realizados levantamentos das necessidades dos trabalhadores frente aos postos de traba1ho. Sendo assim, as mesmas são repassadas ao Serviço Especializado em Engenharia de Segurança e Medicina do Trabalho (SESMT) discutindo sobre propostas de alterações ergonômicas, troca de 
setores e/ou turnos, necessidade de encaminhar o funcionário a alguma especialidade como psicólogo, traumatologista, ginecologista, dentre outros, visando à diminuição do impacto do trabalho e melhorias na qualidade de vida pensando na integralidade da saúde.

\section{VIVÊNCIAS DE RESIDENTES}

A prática multiprofissional se caracteriza pelas diferenças entre as profissões, por meio do entendimento frente ao papel dos profissionais que compõem a equipe, trazendo assim, benefícios em relação a compreensão de cada área, contribuindo também para a integração de conhecimentos e aprendizado do trabalho em equipe (CAFFREY et al., 2011). No grupo formado pelas residentes, é perceptível o espírito de equipe e união, sendo que todas as integrantes conseguem se auxiliar no que for preciso, além de haver um relacionamento interpessoal satisfatório.

Tais conclusões por vezes, também já foram percebidas e destacadas por profissionais integrantes do grupo avaliativo do programa. Acredita-se que tal relação pode ter se estruturado devido que, desde o início da prática o grupo atuou de modo compartilhado, além claro, de ter sido claramente influenciado por características intrínsecas de cada uma como disponibilidade, interesse e cooperação.

Mesmo frente às dificuldades encontradas na atuação, o grupo estabelecido conseguiu trabalhar e otimizar tais situações, uma vez que a troca de saberes e experiências entre as profissionais prevalece. Tais práticas auxiliam cada vez mais para uma prática integral e humanizada, tanto para os pacientes assistidos quanto para as próprias profissionais. Quando a atuação se dá através do trabalho em equipe, é possível perceber a produção de melhores resultados na atenção à saúde - usuários, família e comunidade - além de gerar maiores satisfações no trabalho por parte dos profissionais atuantes (PEDUZZI et al., 2020).

Junto às atividades acima destacadas, é pertinente também salientar realização da elaboração de cartilhas com materiais informativos, voltado para os pacientes e cuidadores, contendo orientações para a alta hospitalar. Os conteúdos abordados tentaram abranger aqueles temas mais vislumbrados durante a prática das residentes, tais como a Diabetes Mellitus (DM), Doença Pulmonar Obstrutiva Crônica (DPOC), pacientes em situação de leito e COVID-19.

Em relação à comunicação entre residentes, instituição de prática e instituição de ensino, pode-se perceber alguns desalinhamentos, o que gerou alguns desconfortos esclarecidos ao longo dos dias de atuação. O início da prática se deu junto ao momento da pandemia da COVID-19, tal situação provocou certa tensão ocorrida entre residentes e gestores das unidades de trabalho pela adequada tomada de decisão para protocolos de atuação prática, coerentes às normas internacionais de proteção a esse novo desafio mundial.

No entanto, umas das legislações que norteiam e se referem à prática atual dos residentes em saúde no país como a Portaria 580 (BRASIL, 2020) que dispõe sobre a Ação Estratégica “O Brasil 
Conta Comigo - Residentes na área de Saúde", para o enfrentamento à pandemia da COVID-19, em seu artigo terceiro - que trata das garantias para que os residentes possam atuar neste contexto - é explicitado o fornecimento de equipamentos de proteção individual aos profissionais de saúde residentes que atuam na contenção do COVID-19 (BRASIL, 2020). Assim como a dificuldade de a instituição de ensino superior ter acesso rápido de aquisição aos EPIs no mercado comercial, no começo destas adaptações de área hospitalar, sendo fornecidos e restabelecidos ao longo do tempo.

A instituição de prática possui o discernimento de que o grupo de residentes tem muito a contribuir com os serviços e demandas que existem no local. Os residentes em saúde, na sua atuação prática possuem a oportunidade de consolidar a integração ensino-serviço, a vista disso, há uma contribuição para os serviços de saúde no conseguimento para o sucesso do cuidado prestado ao indivíduo, permitindo ganho nas situações de saúde dos usuários. Exemplificando esses ganhos pode-se apontar a assistência colaborativa, em favor do cuidado da população em Redes de Atenção à Saúde (MELLO et al., 2018).

\section{DESAFIO DE RESIDENTES}

Em contrapartida ao destaque acima, é notório fatores que diversas vezes impossibilitam a execução de ideias e planejamentos inovadores, como a falta de estrutura física e institucional por parte da organização para dar assistência e colaborar com as atualizações propostas. O que também corrobora para essa percepção é a execução das ações que foram planejadas ao início da residência para o campo de prática, denominado por plano de ação, já que foram encontrados alguns desafios, principalmente quando se refere na necessidade da participação de alguns funcionários da instituição, bem como a necessidade de consideração do fluxo de alguns programas, o que por vezes, impossibilita uma prática efetiva e pontual.

Referente à prática profissional, se torna pertinente destacar que na maioria das vezes as atuações foram realizadas de forma autônoma, permitindo que o residente pudesse experienciar e praticar, possibilitando assim uma aquisição de aprendizagem. Resistências por parte de alguns profissionais do local, em aceitar e considerar as residentes como parte integrante da equipe de trabalho, foram percebidas, mesmo após nove meses de convívio e trabalho em conjunto.

Esse fato pôde ser comprovado em diferentes situações, como no momento de troca de experiências sobre alguns pacientes ou na solicitação de atendimento, sendo na maioria das vezes repassados ao preceptor ao invés da residente responsável pelo caso. Tal característica pode ser percebida no estudo de Alves et al. (2016) onde aborda que os residentes atuantes nas unidades de internação não eram responsáveis diretos pelos pacientes e sim o preceptor de cada área ou de outro profissional do serviço.

Ainda sobre as dificuldades encontradas, no que se refere ao acesso a EPI fornecidos pela instituição, no estudo de Freire et al. (2020) as profissionais residentes referem compartilhar junto à 
equipe do hospital as mudanças e desafios decorrentes da pandemia da COVID-19 como o racionamento de EPI, diferentemente da experiência compartilhada nesse relado onde foi presenciado uma distinção para distribuição destes entre residentes e equipe contratada uma vez que roupas e sapatos apropriados foram disponibilizados somente aos colaboradores.

Outra situação é a possível percepção de que as Residentes estariam ocupando um espaço que não é próprio para equipe de Residência. Um exemplo disso são as reclamações e descontentamentos destacados pelos funcionários da instituição, quando as Residentes estão compartilhando a sala de uso comum de todos das unidades, assim dos computadores para evolução de atendimentos. Desta forma, devido à variedade de núcleos profissionais que se compões o grupo de residentes em Reabilitação Física, as percepções serão divididas por profissional atuante.

\section{Enfermagem}

O campo prático em questão possibilita uma gama de atendimentos para a enfermagem, contribuindo para a realização de diversos procedimentos técnicos quanto à gestão da unidade hospitalar e a interlocução entre a teoria e a prática de atos privativos da enfermagem. Logo no início das atividades práticas foi vivenciada a resistência por parte de alguns funcionários do hospital quanto à visualização da residente enfermeira como membro líder da equipe, porém este obstáculo logo foi superado ao longo das práticas.

De modo geral, a equipe de funcionários se mostra flexível, manifestando espírito cooperativo de equipe. No decorrer da prática, a grande maioria das rotinas da unidade eram realizadas com autonomia, não necessitando de uma supervisão direta por parte da preceptora. Quanto à equipe de residentes há um bom relacionamento interpessoal entre todas as integrantes, possibilitando por diversos momentos o compartilhamento de informações sobre vivências das atividades realizadas. O que contribuiu para a troca de saberes entre as mesmas.

\section{Fisioterapia}

Por vezes, a residente não se sente acolhida, uma vez que vê sua opinião sendo desconsiderada no que se refere às condutas fisioterapêuticas a serem realizadas junto aos pacientes internados, muito em decorrência da resistência ou desconhecimento por parte de alguns profissionais do corpo médico que não permitem que o mesmo possua autonomia enquanto profissional já formado. Essa dificuldade de compartilhamento não ocorre somente com o residente, mas também com outros núcleos da equipe. Diante da constante visão do residente como acadêmico/ estagiário, ainda assim é notável o direcionamento da equipe ao preceptor - trabalhador do hospital, para conferência dos atendimentos, os quais possuem maior reconhecimento em seu ambiente de trabalho. 
Devido à boa convivência com a equipe em geral, oportunizou-se a troca de informações sobre os atendimentos, visto que a equipe recorre aos residentes constantemente para avaliação dos pacientes, evidenciando a importância dos mesmos como integrantes da equipe, assim como a viabilização de atendimentos compartilhados favorecendo a multiprofissionalidade e interterdisciplinaridade. A inserção nos rounds multiprofissionais é vista como um ganho para a residência, uma vez que nele se dá um espaço propicio para trocas, podendo assim expor argumentos enquanto profissional não médico, podendo elencar prioridades terapêuticas considerando a integralidade do paciente.

\section{Nutrição}

No decorrer da prática, percebeu-se uma autonomia profissional enquanto núcleo de nutrição. Sendo a residente responsável pela avaliação antropométrica e prescrição da dieta adequada conforme a necessidade do paciente internado, em especial os que apresentam intervenções e necessitam se nutrir via Sonda Nasoentérica.

Sobre os desafios da prática clínica, pode-se destacar a dificuldade de comunicação com alguns médicos responsáveis pelos pacientes na unidade de internação, posto que não são todos que se mostram disponíveis ao diálogo interprofissional. Mesmo na presença de uma visão acadêmica sobre o profissional residente, com a convivência diária, galgou-se um relacionamento afetivo com as técnicas de enfermagem e ao mesmo tempo efetivo, uma vez que estas reconhecem o trabalho da nutricionista na unidade, recorrendo a esta sobre suas dúvidas e solicitações de orientação sobre a dieta administrada.

No que tange a atuação no projeto, a sensação que se tem é de desprestígio por acreditar que não está sendo valorizada, uma vez que a instituição hospitalar, por vezes, se mostra inflexível. Nota-se que apenas há retorno à equipe de residentes quando a instituição acredita ser pertinente, desconsiderando as necessidades elencadas pelas residentes. Por fim, a residente de nutrição mantém um bom relacionamento interpessoal com a toda equipe, seja esta da unidade de internação clínica como médicos, enfermeiras e técnicas de enfermagem, bem como com a equipe de residentes, acreditando estar inserida em ambas e contribuindo para uma prática de relações integradas e horizontais.

\section{Psicologia}

O campo hospitalar é considerado um campo potencialmente rico para aquisição de conhecimento, bem como assimilação entre teoria e prática, além de propiciar uma atuação junto a uma equipe multiprofissional, prática muitas vezes não incentivada na formação em Psicologia. Além disso, possibilita inúmeras trocas e discussões entre os núcleos de saberes, assim como proporciona o desenvolvimento de manejo clínico durante os atendimentos no hospital. 
Em contrapartida, percebem-se algumas limitações referentes à eficácia de certos processos e procedimentos, muitas vezes por consequência de centralização de poder, tanto no que se refere ao campo de saber, mas também em relação à administração do local. Em relação à parte teórica da especialização, percebem-se algumas falhas quando se trata da abrangência de todos os núcleos participantes do programa, uma vez que na grade curricular não se tem docentes da área da Psicologia, além de muitas vezes não ser abordados todos os materiais necessários para atuação, o que demanda para o residente a necessidade de buscas além do que é ofertado pela residência.

Coordenador, tutor e preceptor se mostram acessíveis, possibilitam um espaço de trocas e colocações. Por certos momentos, são notáveis algumas inflexibilidades por parte do programa de residência, o que leva a pensar por vezes na ausência de um olhar humanizado para os profissionais atuantes, resultando muitas vezes no sentimento de desamparo. É notável que o local esteja em processo de mudança e atualizações, o que pode resultar em benefícios para todos os envolvidos no serviço. Frente a tal fato, é perceptível a grande demanda dessa inovação depositada no residente, em diversas situações requisitando destes, ideias e planejamentos de ações que muitas vezes, poderiam ser realizadas pelos servidores do local.

\section{Terapia Ocupacional}

O maior desafio encontrado enquanto núcleo de Terapia Ocupacional é certamente o desconhecimento da equipe de saúde sobre o objeto de estudo e o papel desta profissão especialmente no ambiente hospitalar, podendo este entrave estar atrelado à ausência deste profissional contratado na instituição em questão. No decorrer dos seis meses de atividade prática neste campo, a residente foi construindo essa identidade paulatinamente, assim como o restante da equipe se apropriando sobre as possíveis intervenções a serem realizadas pelo terapeuta ocupacional. Acredita-se que isso se deva aos atendimentos multiprofissionais e as trocas constantes na unidade de internação clínica, havendo maiores solicitações por parte dos profissionais no decorrer dos meses de atuação.

A ambiguidade em relação à consideração do residente como parte integrante da equipe é outro desafio a ser superado, uma vez que para muitas atividades como a execução de tarefas este é considerado, porém para tomada de decisões não. No que tange algumas questões, como a realização da contrarreferência de pacientes que estão por receber alta, a residência é a única referência em todo serviço, fato que pode as considerar como integrante da equipe; mas quando ocorrem reuniões internas a respeito da organização do serviço estas não são incluídas.

\section{CONCLUSÃO}

Frente às atividades práticas prevaleceram a troca de saberes e experiências entre as residentes, mesmo diante das dificuldades encontradas no campo de atuação. Os atendimentos foram realizados 
de forma integral e humanizada, tanto para a assistência prestada aos usuários quanto para as próprias residentes. Em virtude ao que foi mencionado no estudo é perceptível a importância da criação de um senso crítico diante dos desafios vivenciados na etapa formativa de especialização, tendo em vista uma maior preparação para uma futura atuação profissional, pois estes desafios proporcionam uma visão mais efetiva do campo de atuação.

Os enfrentamentos pelos quais o grupo em questão passou quer seja em esferas pessoais, profissionais e institucionais, foram aprendizados para superar os obstáculos ligados ao acolhimento e as trocas entre demais profissionais, já que, por vezes, estas experienciaram resistências e objeções frente ao seu papel enquanto profissional atuante. Bem como foram aos poucos ganhando maior saber do profissional residente, influenciadas pela boa estrutura institucional em sua organização e a demonstração de sua bagagem de experiências aqui apreendidas. Também ressaltaram a necessidade de contratação de equipes especializadas pela própria instituição, efetivando-se belas intervenções proporcionadas aos usuários e serviços.

Conclui-se ainda que a inserção e a participação mais ativa dos residentes nas ações e na equipe do setor em que atuam, pode contribuir melhor na construção coletiva de conhecimentos e efetividade profissional. Assim sendo, o residente cada vez mais inserido nos processos da equipe e evidenciando suas potencialidades frente ao campo de atuação se afirma enquanto profissional capaz de uma prática com autonomia.

\section{REFERÊNCIAS}

ALVES, C.C. et al. Relato de experiência da atuação do nutricionista em Residência Multiprofissional em Saúde. Revista de Nutrição, Campinas, v. 29, n. 4, p. 597-608, 2016.

ANASTASIOU, L.G.C.; ALVES L.P. Processos de ensinagem na universidade: pressupostos para as estratégias de trabalho em aula. Joinville: Ed. Univille; 2003.

BRASIL. Lei no 11.129, de 30 de junho de 2005. Institui a Residência em Área Profissional de Saúde e cria a Comissão Nacional de Residência Multiprofissional em Saúde CNRMS. Brasilia, DF, 2005.

BRASIL. Ministério da Saúde. Conselho Nacional de Saúde. Resolução no 510, de 7 de abril de 2016. Trata sobre as diretrizes e normas regulamentadoras de pesquisa em ciências humanas e sociais. Diário Oficial da União, Brasília, DF, 2016. 
BRASIL. Diário Oficial da União. Portaria n⿳ 580, de 27 de março de 2020. Dispõe sobre a Ação Estratégica "O Brasil Conta Comigo - Residentes na área de Saúde", para o enfrentamento à pandemia do coronavírus (COVID-19). Brasilia, DF, 2020.

CAFFREY, R. G. et al. An educational program to promote positive communication and collaboration between nurses and medical staff. Journal for Nurses in Professional Development, v. 27, n. 3, p. 121-127, 2011.

COMISSÃO NACIONAL DE RESIDÊNCIA MULTIPROFISSIONAL EM SAÚDE. Resolução CNRMS no 5, de 7 de novembro de 2014. Diário Oficial da União; Poder Executivo, Brasília, DF Nº 217, 10 de novembro de 2014. Seção I, p.34.

FREIRE, M.S.F. et al. Profissional residente no enfrentamento da COVID-19: relato de experiência no contexto da enfermagem intensiva. Enfermagem Brasil, Brasil, v. 19, n. 4, p. 13-20, 2020.

MARTINS, G.D.M. et al. Implementação de residência multiprofissional em saúde de uma universidade federal: trajetória histórica. Revista Gaúcha de Enfermagem, Porto Alegre, v. 37, n. 3, 2016.

MELLO, A. L. et al. Formação de residentes multiprofissionais em saúde: limites e contribuições para a integração ensino-serviço. Revista de Enfermagem do Centro-Oeste Mineiro, Minas Gerais, v. 8, 2018.

PEDUZZI, M. et al. Trabalho em equipe: uma revisita ao conceito e a seus desdobramentos no trabalho interprofissional. Trabalho, Educação e Saúde, Rio de Janeiro, v. 18, 2020.

ROSSIT, R.; BATISTA, S.H.; BATISTA, N.A. Formação para a integralidade no cuidado: potencialidades de um projeto interprofissional. Revista internacional de humanidades médicas, São Paulo, v. 3, n. 1, 2014.

SILVA, C.T. et al. Residência multiprofissional como espaço intercessor para a educação permanente em saúde. Texto \& Contexto-Enfermagem, v. 25, n. 1, 2016.

SILVA, L.B. Residência Multiprofissional em Saúde no Brasil: alguns aspectos da trajetória histórica. Revista Katálysis, Florianópolis, v. 21, n. 1, p. 200-209, 2018. 
SILVA, L.C.; GOMES, M.R.B. Residência multiprofissional em saúde: desafios e possibilidades para o serviço social. Revista Serviço Social em Perspectiva - Montes Claros/MG, v. 2, n. 2, jul/dez-2018. p. $107-128$.

SILVA, R.M.B.; MOREIRA, S.N.T. Estresse e Residência Multiprofissional em Saúde: Compreendendo Significados no Processo de Formação. Revista Brasileira de Educação Médica, v. 43, n. 4, p. 157-166, 2019.

UNIVERSIDADE FRANCISCANA. Programa de Residência em Reabilitação Física. Processo de Seleção de Programa de Residência em Área Profissional da Saúde - Reabilitação, 2015. 